\title{
A projective splitting algorithm for solving generalized mixed variational inequalities
}

Fu-quan $\mathrm{Xia}^{1 *}$ and Yun-zhi Zou ${ }^{2}$

\author{
* Correspondence: fuquanxia@sina. \\ com \\ 'Department of Mathematics, \\ Sichuan Normal University, \\ Chengdu, Sichuan 610066, P. R. \\ China \\ Full list of author information is \\ available at the end of the article
}

\begin{abstract}
In this paper, a projective splitting method for solving a class of generalized mixed variational inequalities is considered in Hilbert spaces. We investigate a general iterative algorithm, which consists of a splitting proximal point step followed by a suitable orthogonal projection onto a hyperplane. Moreover, in our splitting algorithm, we only use the individual resolvent mapping $\left(I+\mu_{k} \partial f^{-1}\right.$ and never work directly with the operator $T+\partial f$, where $\mu_{k}$ is a positive real number, $T$ is a set-valued mapping and $\partial f$ is the sub-differential of function $f$. We also prove the convergence of the algorithm for the case that $T$ is a pseudomonotone set-valued mapping and $f$ is a non-smooth convex function.
\end{abstract}

2000 Mathematics Subject Classification: 90C25; 49D45; 49D37.

Keywords: projective splitting method, generalized mixed variational inequality, pseudomonotonicity

\section{Introduction}

Let $X$ be a nonempty closed convex subset of a real Hilbert space $H, T: X \rightarrow 2^{H}$ be a set-valued mapping and $f: H \rightarrow(-\infty,+\infty]$ be a lower semi-continuous (1.s.c) proper convex function. We consider a generalized mixed variational inequality problem (GMVIP): find $x^{*} \in X$ such that there exists $w^{*} \in T\left(x^{*}\right)$ satisfying

$$
\left\langle w^{*}, y-x^{*}\right\rangle+f(y)-f\left(x^{*}\right) \geq 0, \quad \forall y \in X .
$$

The GMVIP (1.1) has enormous applications in many areas such as mechanics, optimization, equilibrium, etc. For details, we refer to [1-3] and the references therein. It has therefore been widely studies by many authors recently. For example, by Rockafellar [4], Tseng [5], Xia and Huang [6] and the special case $(f=0)$ was studied by Crouzeix [7], Danniilidis and Hadjisavvas [8] and Yao [9].

A large variety of problems are special instances of the problem (1.1). For example, if $T$ is the sub-differential of a finite-valued convex continuous function $\phi$ defined on Hilbert space $H$, then the problem (1.1) reduces to the following non-differentiable convex optimization problem:

$$
\min _{x \in X}\{f(x)+\varphi(x)\} .
$$

Furthermore, if $T$ is single-valued and $f=0$, then the problem (1.1) reduces to the following classical variational inequality problem: find $x^{*} \in X$ such that, for all $y \in X$, 


$$
\left\langle T\left(x^{*}\right), y-x^{*}\right\rangle \geq 0 \text {. }
$$

Many methods have been proposed to solve classical variational inequalities (1.2) in finite and infinite dimensional spaces. The simple one among these is the projection method which has been intensively studied by many authors (see, e.g., [10-14]). However, the classical projection method does not work for solving the GMVIP (1.1). Therefore, it is worth studying other implementable methods for solving the problem (1.1).

Algorithms that can be applied for solving the problem (1.1) or one of its variants are very numerous. For the case when $T$ is maximal monotone, the most famous method is the proximal method (see, e.g., Rockafellar [4]). Splitting methods have also been studied to solve the problem (1.1). Here, the set-valued mapping $T$ and $\partial\left(f+\psi_{X}\right)$ play separate roles, where $\psi_{X}$ denotes the indicator function associated with $X$ (i.e., $\psi_{X}$ $(x)=0$ if $x \in X$ and $+\infty$ otherwise) and $\partial\left(f+\psi_{X}\right)$ denotes the sub-differential of the convex function $f+\psi_{X}$. The simplest splitting method is the forward-backward scheme (see, e.g., Tseng [5]), in which the iteration is given by

$$
x^{k+1} \in\left[I+\mu_{k} \partial\left(f+\psi_{X}\right)\right]^{-1}\left[I-\mu_{k} T\right]\left(x^{k}\right),
$$

where $\left\{\mu_{k}\right\}$ is a sequence of positive real numbers. Cohen [15] developed a general algorithm framework for solving the problem (1.1) in Hilbert space $H$, based on the so-called auxiliary problem principle. The corresponding method is a generalization of the forward-backward method. Due to the auxiliary problem principle Cohen [15], Salmon et al. [16] developed a bundle method for solving the problem (1.1).

For solving the GMVIP (1.1), some authors assumed that $T$ is upper semi-continuous and mono-tone(or some other stronger conditions, e.g., strictly monotone, paramonotone, maximal monotone, strongly monotone). Moreover, their methods fail to provide convergence under weaker conditions than the monotonicity of $T$. So, it is a significant work that how to solve the problem (1.1) when $T$ fails to be monotone. This is one of the main motivations of this paper.

On the other hand, the GMVIP (1.1) can be expressed as an inclusion form as follows: find $x^{*} \in X$ such that

$$
0 \in T\left(x^{*}\right)+\partial\left(f+\psi_{X}\right)\left(x^{*}\right)
$$

Thus, the problem (1.1) is a special case of the following inclusion problem:

$$
0 \in A(x)+B(x)
$$

where $A$ and $B$ are set-valued operators on real Hilbert space $H$.

The algorithms for solving the inclusion (1.4) have an extensive literature. The simplest one among these is the splitting method. All splitting methods can be essentially divided into three classes: Douglas/Peaceman-Rachford class (see, e.g., [17,18]), the double-backward class (see, e.g., [19]), and the forward-backward class (see, e.g., $[20,21])$. Therefore, one natural problem is whether the splitting method can be developed for solving (1.1). This is another main motivation of this paper.

In this paper, we provide a projective splitting method for solving the GMVIP (1.1) in Hilbert spaces. Our iterative algorithm consists of two steps. The first step of the algorithm in generating a hyperplane separating $z_{k}$ from the solution set of problem (1.1). The second step is then to project $z_{k}$ onto this hyperplane (with some relaxation 
factor). We first prove that the sequences $\left\{x^{k}\right\}$ and $\left\{z^{k}\right\}$ are weakly convergent. We also prove that the weak limit point of $\left\{x^{k}\right\}$ is the same as the weak limit point of $\left\{z^{k}\right\}$. Moreover, we obtain that the weak limit point of these sequences is a solution of the problem (1.1) under the conditions that the set-valued mapping $T$ is pseudomonotone with respect to $f$ and the function $f$ is convex.

\section{Preliminaries}

For a convex function $f: H \rightarrow(-\infty,+\infty]$, let $\operatorname{dom} f=\{x \in H: f(x)<\infty\}$ denote its effective domain, and let

$$
\partial f(\cdot)=\{p \in H: f(y) \geq f(\cdot)+\langle p, y-\cdot\rangle, \quad \forall y \in H\}
$$

denote its sub-differential.

Suppose that $X \subset H$ is a nonempty closed convex subset and

$$
\operatorname{dist}(z, X):=\inf _{x \in X}\|z-x\|
$$

is the distance from $z$ to $X$. Let $P_{X}[z]$ denote the projection of $z$ onto $X$, that is, $P_{X}[z]$ satisfies the condition

$$
\left\|z-P_{X}[z]\right\|=\operatorname{dist}(z, X) .
$$

The following well-known properties of the projection operator will be used later in this paper.

Proposition 2.1. [22] Let $X$ be a nonempty closed convex subset in $H$, the following properties hold:

(i) $\left\langle x-y, x-P_{X}[x]\right\rangle \geq 0$, for all $x \in H$ and $y \in X$;

(ii) $\left\langle P_{X}[x]-x, y-P_{X}[x]\right\rangle \geq 0$, for all $x \in H$ and $y \in X$;

(iii) $\left\|P_{X}[x]-P_{X}[y]\right\| \leq\|x-y\|$, for all $x, y \in H$.

Definition 2.1. Let $X$ be a nonempty subset of a Hilbert space $H$, and let $f: X \rightarrow$ $(-\infty,+\infty]$ a function. A set-valued mapping $T: X \rightarrow 2^{H}$ is said to be

(i) monotone if

$$
\langle u-v, x-y\rangle \geq 0, \quad \forall x, y \in X, u \in T(x), v \in T(y) ;
$$

(ii) pseudomonotone with respect to $f$ if for any $x, y \in X, u \in T(x), v \in T(y)$,

$$
\langle u, y-x\rangle+f(y)-f(x) \geq 0 \Rightarrow\langle v, y-x\rangle+f(y)-f(x) \geq 0 .
$$

We will use the following Lemmas.

Lemma 2.1. [23] Let $D$ be a nonempty convex set of a topological vector space $E$ and let $\varphi: D \times D \rightarrow \mathbb{R} \cup\{+\infty\}$ be a function such that

(i) for each $v \in D, u \rightarrow \varphi(v, u)$ is upper semi-continuous on each nonempty compact subset of $D$;

(ii) for each nonempty finite set $\left\{v_{1}, \cdots, \cdot v_{m}\right\} \subset D$ and for each $u=\sum_{i=1}^{m} \lambda_{i} v_{i}\left(\lambda_{i} \geq 0, \Sigma_{i=1}^{m} \lambda_{i}=1\right), \max _{1 \leq i \leq m} \varphi\left(v_{i}, u\right) \geq 0$; 
(iii) there exists a nonempty compact convex subset $D_{0}$ of $D$ and a nonempty compact subset $K$ of $D$ such that, for each $u \in D \backslash K$, there is $v \in \operatorname{co}\left(D_{0} \cup\{u\}\right)$ with $\varphi(v, u)<0$.

Then, there exists $\hat{u} \in K$ such that $\phi(v, \hat{u}) \geq 0$ for all $v \in D$.

Lemma 2.2. [24, p. 119] Let $X, Y$ be two topological spaces, $W: X \times Y \rightarrow \mathbb{R}$ be an upper semi-continuous function, and $G: X \rightarrow 2^{Y}$ be upper semi-continuous at $x_{0}$ such that $G\left(x_{0}\right)$ is compact. Then, the marginal function $V$ defined on $X$ by

$$
V(x)=\sup _{y \in G(x)} W(x, y)
$$

is upper semi-continuous at $x_{0}$.

Lemma 2.3. [25] Let $\sigma \in[0,1)$ and $\mu=\sqrt{1-\left(1-\sigma^{2}\right)^{2}}$. If $v=u+\xi$, where $\|\xi\|^{2} \leq$ $\sigma^{2}\left(\|u\|^{2}+\|v\|^{2}\right)$, then

(i) $\langle u, v\rangle \geq\left(\|u\|^{2}+\|v\|^{2}\right)\left(1-\sigma^{2}\right) / 2$;

(ii) $(1-\mu)\|v\| \leq\left(1-\sigma^{2}\right)\|u\| \leq(1+\mu)\|v\|$.

\section{Projective splitting method}

$\psi_{X}: H \rightarrow(-\infty,+\infty]$ be the indicator function associated with $X$. Choose three positive sequences $\left\{\lambda_{k}>0\right\},\left\{\alpha_{k}\right\} \in(0,2)$ and $\left\{\rho_{k}\right\} \in(0,2)$. Select a fixed relative error tolerance $\sigma \in[0,1)$. We first describe a new projective splitting algorithm for the GMVIP (1.1), and then give some preliminary results on the algorithm.

\section{Algorithm 3.1.}

Step 0. (Initiation) Select initial $z^{0} \in X$. Set $k=0$.

Step 1. (Splitting proximal step) Find $x^{k} \in X$ such that

$$
\begin{aligned}
& x^{k}+\lambda_{k} g^{k}=z^{k}+\lambda_{k} \xi^{k}, \quad g^{k} \in \partial\left[f+\psi_{X}\right]\left(x^{k}\right) \\
& x^{k}-\lambda_{k} w^{k}=\left(1-\alpha_{k}\right) z^{k}+\alpha_{k} x^{k}-\lambda_{k} \xi^{k}, \quad w^{k} \in T\left(x^{k}\right)
\end{aligned}
$$

where the residue $\xi^{k} \in H$ is required to satisfy the following condition:

$$
\left\|\xi^{k}\right\| \leq \sigma \sqrt{\alpha_{k}^{2}\left\|z^{k}-x^{k}\right\|^{2} /\left(4 \lambda_{k}^{2}\right)+\left\|g^{k}+w^{k}\right\|^{2}} / 4
$$

Step 2. (Projection step) If $g^{k}+w^{k}=0$, then STOP; otherwise, take

$$
\bar{z}^{k}=z^{k}-\beta_{k}\left(g^{k}+w^{k}\right) \text { with } \beta_{k}=\left\langle g^{k}+w^{k}, z^{k}-x^{k}\right\rangle /\left\|g^{k}+w^{k}\right\|^{2} .
$$

Step 3. Set $z^{k+1}=z^{k}+\rho_{k}\left(\bar{z}^{k}-z^{k}\right)$.

Step 4. Let $k=k+1$ and return to Step 1 .

In this paper, we focus our attention on obtaining general conditions ensuring the convergence of $\left\{z^{k}\right\}_{k \in N}$ and $\left\{x^{k}\right\}_{k \in N}$ toward a solution of problem (1.1), under the following hypotheses on the parameters:

$$
\lambda_{1}:=\inf _{k \geq 0} \lambda_{k}>0, \quad \lambda_{2}:=\sup _{k \geq 0} \lambda_{k}<\infty,
$$




$$
R_{1}:=\inf _{k \geq 0} \rho_{k}>0 \quad \text { and } \quad R_{2}:=\sup _{k \geq 0} \rho_{k}<2,
$$

To motivate Algorithm 3.1, we note that (3.1) implies $x^{k}=\left(I+\lambda_{k} \partial f\right)^{-1}\left(z^{k}+\lambda_{k} \xi_{k}\right)$, and that the operator $\left(I+\lambda_{k} \partial f\right)^{-1}$ is everywhere defined and single-valued. Rearranging (3.1) and (3.2), one has $g^{k}=\xi^{k}+\frac{z^{k}-x^{k}}{\lambda_{k}}$ and $w^{k}=\frac{1-\alpha_{k}}{\lambda_{k}}\left(x^{k}-z^{k}\right)+\xi^{k}$. Algorithm 3.1 is a true splitting method for problem (1.1), in that it only uses the individual resolvent mapping $\left(I+\lambda_{k} \partial f\right)^{-1}$, and never works directly with the operator $\partial f+T$. The existence of $x^{k} \in X$ and $w^{k} \in T\left(x^{k}\right)$ such that (3.1)-(3.2) will be proved in the following Theorem 3.1.

Substituting (3.1) into (3.2) and simplifying, we obtain

$$
\alpha_{k}\left(x^{k}-z^{k}\right) / \lambda_{k}+g^{k}+w^{k}=2 \xi^{k} .
$$

This method is the so-called inexact hybrid proximal algorithm for solving problem (1.1). Obvious that problem (3.7) is solved only approximately and the residue $\xi^{k} \in H$ satisfying (3.3). There are at least two reasons for dealing with the proximal algorithm (3.7). First, it is generally impossible to find an exact value for $x^{k}$ given by (3.1) and (3.2). Particularly when $T$ is nonlinear; second, it is clearly inefficient to spend too much effort on the computation of a given iterate $z^{k}$ when only the limit of the sequence $\left\{x^{k}\right\}$ has the desired properties.

It is easy to see that (3.4) is a projection step because it can be written as $\bar{z}^{k}=P_{K}\left(z^{k}\right)$, where $P_{K}: H \rightarrow K$ is the orthogonal projection operator onto the half-space $K=\{z \in$ $\left.H:\left\langle g^{k}+w^{k}, z-x^{k}\right\rangle \leq 0\right\}$. In fact, by (3.4) we have $\bar{z}^{k}=z^{k}-\beta_{k}\left(g^{k}+w^{k}\right)$. Then for each $y \in K$, we deduce that

$$
\begin{aligned}
& \left\langle z^{k}-\bar{z}^{k}, y-z^{k}\right\rangle=\beta_{k}\left\langle g^{k}+w^{k}, y-z^{k}\right\rangle \\
& =\beta_{k}\left\langle g^{k}+w^{k}, y-x^{k}\right\rangle+\beta_{k}\left\langle g^{k}+w^{k}, x^{k}-z^{k}\right\rangle \\
& =\beta_{k}\left\langle g^{k}+w^{k}, x^{k}-z^{k}\right\rangle\left(\text { since }\left\langle g^{k}+w^{k}, y-x^{k}\right\rangle \leq 0\right) \\
& \leq 0\left(\text { since } \beta_{k}=\left\langle g^{k}+w^{k}, z^{k}-x^{k}\right\rangle /\left\|g^{k}+w^{k}\right\|^{2}\right) .
\end{aligned}
$$

By Proposition 2.1, we know that $\bar{z}^{k}=P_{K}\left(z^{k}\right)$. By pseudomonotonicity of $T$ with respect to $f$ and Theorem 4.1(ii) below, the hyperplane $K$ separates the current iterate $z^{k}$ from the set $S=\{x \in H: 0 \in \partial f(x)+T(x)\}$. Thus, in Algorithm 3.1, the splitting proximal iteration is used to construct this separation hyperplane, the next iterate $z^{k+1}$ is then obtained by a trivial projection of $z^{k}$, which is not expensive at all from a numerical point of view.

Now, we will prove that the sequence $\left\{x^{k}\right\}$ is well defined and so is the sequence $\left\{z^{k}\right\}$. Note that if $x^{k}$ satisfies (3.1)-(3.2) together with (3.3), with $\sigma=0$, then $x^{k}$ always satisfies these conditions with any $\sigma \in[0,1)$. Since $\sigma=0$ also implies that the error term $\xi^{k}$ vanishes, existence of $x^{k}$ for $\xi^{k}=0$ is enough to ensure the existence of $\xi^{k} \neq 0$. So in the following theorem 3.1, we assume that $\xi^{k}=0$.

Theorem 3.1. Let $X$ be a nonempty closed convex subset of a Hilbert space $H$, and let $f: X \rightarrow(-\infty,+\infty]$ be a l.s.c proper convex function. Assume that $T: X \rightarrow 2^{H}$ is pseudomonotone with respect to $f$ and upper semi-continuous from the weak topology to the weak topology with weakly compact convex values. If the parameter $\alpha_{k}, \lambda_{k}>0$ 
and solution set of problem (1.1) is nonempty, then for each given $z^{k} \in X$, there exist $x^{k} \in X$ and $w^{k} \in T\left(x^{k}\right)$ satisfying (3.1)-(3.2).

Proof. For each given $z^{k} \in X$ and $\xi^{k}=0$, it follows from (3.1) and (3.2) that,

$$
g^{k}=\frac{1}{\lambda_{k}}\left[\alpha_{k}\left(z^{k}-x^{k}\right)-\lambda_{k} w^{k}\right]
$$

where $g^{k} \in \partial\left[f+\psi_{X}\right]\left(x^{k}\right)$ and $w^{k} \in T\left(x^{k}\right)$. (3.8) is equivalent to the following inequality:

$$
\alpha_{k} \lambda_{k}^{-1}\left\langle x^{k}-z^{k}, y-x^{k}\right\rangle+\left\langle w^{k}, y-x^{k}\right\rangle+f(y)-f\left(x^{k}\right) \geq 0, \quad \forall y \in X .
$$

So we consider the following variational inequality problem: find $x^{k} \in X$ such that for each $y \in X$,

$$
\alpha_{k} \lambda_{k}^{-1}\left\langle x^{k}-z^{k}, y-x^{k}\right\rangle+\sup _{w^{k} \in T\left(x^{k}\right)}\left\langle w^{k}, y-x^{k}\right\rangle+f(y)-f\left(x^{k}\right) \geq 0 .
$$

For the sake of simplicity, we rewrite the problem (3.9) as follows: find $\bar{x} \in X$ such that

$$
\alpha_{k} \lambda_{k}^{-1}\left\langle\bar{x}-z^{k}, y-\bar{x}\right\rangle+\sup _{w \in T(\bar{x})}\langle w, y-\bar{x}\rangle+f(y)-f(\bar{x}) \geq 0, \quad \forall y \in X .
$$

For each fixed $k$, define $\varphi: X \times X \rightarrow(-\infty,+\infty]$ by

$$
\phi(y, x)=\alpha_{k} \lambda_{k}^{-1}\left\langle x-z^{k}, y-x\right\rangle+\sup _{w \in T(x)}\langle w, y-x\rangle+f(y)-f(x) .
$$

Since $T$ is upper semi-continuous from the weak topology to weak topology with weakly compact values, by Lemma 2.2, we know that the mapping $V(x)=\sup _{w \in T(x)}\langle w, y-x\rangle$ is upper semi-continuous from the weak topology to weak topology. Noting that $f$ is a l.s.c convex function, for each $y \in X$, the function $x \alpha \varphi(y, x)$ is weakly upper semi-continuous on $X$. We now claim that $\varphi(y, x)$ satisfies condition (ii) of Lemma 2.1. If it is not, then there exists a finite subset $\left\{y^{1}, y^{2}, \ldots, y^{m}\right\}$ of $X$ and $x=\Sigma_{i=1}^{m} \delta_{i} y^{i}\left(\delta_{i} \geq 0, i=1,2, \ldots, m\right.$ with $\left.\sum_{i=1}^{m} \delta_{i}=1\right)$ such that $\varphi\left(y^{i}, x\right)<0$ for all $i=1,2, \cdots, m$. Thus,

$$
\alpha_{k} \lambda_{k}^{-1}\left\langle x-z^{k}, y^{i}-x\right\rangle+\sup _{w \in T(x)}\left\langle w, y^{i}-x\right\rangle+f\left(y^{i}\right)-f(x)<0, \quad \forall i=1,2, \cdots, m
$$

and so

$$
\alpha_{k} \lambda_{k}^{-1} \sum_{i=1}^{m} \delta_{i}\left\langle x-z^{k}, y^{i}-x\right\rangle+\sum_{i=1}^{m} \delta_{i} \sup _{w \in T(x)}\left\langle w, y^{i}-x\right\rangle+\sum_{i=1}^{m} \delta_{i}\left[f\left(y^{i}\right)-f(x)\right]<0 .
$$

By the convexity of $f$, we get

$$
0=\alpha_{k} \lambda_{k}^{-1}\left\langle x-z^{k}, x-x\right\rangle+\sup _{w \in T(x)}\langle w, x-x\rangle<0,
$$

which is a contradiction. Hence, condition (ii) of Lemma 2.1 holds.

Now, let $\hat{y} \in X$ be a solution of problem (1.1). Then, there exists $\hat{w} \in T(\hat{y})$ such that

$$
\langle\hat{w}, x-\hat{y}\rangle+f(x)-f(\hat{y}) \geq 0, \quad \forall x \in X .
$$


By the pseudomonotonicity of $T$ with respect to $f$, for all $x \in X$,

$$
\langle w, \hat{y}-x\rangle+f(\hat{y})-f(x) \leq 0, \quad \forall w \in T(x),
$$

and so

$$
\sup _{w \in T(x)}\langle w, \hat{y}-x\rangle+f(\hat{y})-f(x) \leq 0, \quad \forall x \in X
$$

On the other hand, we have

$$
\begin{aligned}
\phi(\hat{y}, x)= & \alpha_{k} \lambda_{k}^{-1}\left\langle x-z^{k}, \hat{y}-x\right\rangle+\sup _{w \in T(x)}\langle w, \hat{y}-x\rangle+f(\hat{y})-f(x) \\
\leq & \alpha_{k} \lambda_{k}^{-1}\langle x-\hat{y}, \hat{y}-x\rangle+\alpha_{k} \lambda_{k}^{-1}\left\langle\hat{y}-z^{k}, \hat{y}-x\right\rangle \\
& +\sup _{w \in T(x)}\langle w, \hat{y}-x\rangle+f(\hat{y})-f(x) \\
\leq \quad & -\alpha_{k} \lambda_{k}^{-1}|| x-\hat{y}\left\|^{2}+\alpha_{k} \lambda_{k}^{-1}\left(\|\hat{y}\|+\left\|z^{k}\right\|\right)\right\| x-\hat{y} \| \\
& +\sup _{w \in T(x)}\langle w, \hat{y}-x\rangle+f(\hat{y})-f(x) .
\end{aligned}
$$

We consider the following equation in $\mathbb{R}$ :

$$
-\alpha_{k} \lambda_{k}^{-1} x^{2}+\alpha_{k} \lambda_{k}^{-1}\left(\|\hat{y}\|+\left\|z^{k}\right\|\right) x=0 .
$$

It is obviously that equation (3.12) has only one positive solution $r=\|\hat{y}\|+\left\|z^{k}\right\|$. If the real number $x>r$, we have

$$
-\alpha_{k} \lambda_{k}^{-1} x^{2}+\alpha_{k} \lambda_{k}^{-1}\left(\|\hat{y}\|+\| z^{k}||\right) x<0 .
$$

Thus, when $\|x-\hat{y}\|>r$, we obtain

$$
-\alpha_{k} \lambda_{k}^{-1}\|x-\hat{y}\|^{2}+\alpha_{k} \lambda_{k}^{-1}\left(\|\hat{y}\|+\left\|z^{k}\right\|\right)\|x-\hat{y}\|<0 .
$$

Let

$$
X_{0}=\{x \in H:\|\hat{y}-x\| \leq r\} .
$$

Then, $D_{0}=\{\hat{y}\}$ and $X_{0}$ are both weakly compact convex subsets of Hilbert space $H$. By (3.11) and (3.13), we deduce that for each $x \in X \backslash X_{0}$, there exists a $\hat{y} \in \operatorname{co}\left(D_{0} \cup\{x\}\right)$ such that $\phi(\hat{y}, x)<0$. Hence, all conditions of Lemma 2.1 are satisfied. Now, Lemma 2.1 implies that there exists a $\bar{x} \in X$ such that $\phi(y, \bar{x}) \geq 0$ for all $y \in X$. That is,

$$
\alpha_{k} \lambda_{k}^{-1}\left\langle\bar{x}-z^{k}, y-\bar{x}\right\rangle+\sup _{w \in T(\bar{x})}\langle w, y-\bar{x}\rangle+f(y)-f(\bar{x}) \geq 0, \quad \forall y \in X .
$$

Therefore, $x^{k}=\bar{x} \in X$ is a solution of the problem (3.9). By the assumptions on $T$, we know that there exists $w^{k} \in T\left(x^{k}\right)$ such that

$$
\alpha_{k} \lambda_{k}^{-1}\left\langle x^{k}-z^{k}, y-x_{\rho}^{k}\right\rangle+\left\langle w^{k}, y-x^{k}\right\rangle+f(y)-f\left(x^{k}\right) \geq 0, \quad \forall y \in X
$$

Thus, $x^{k} \in X$ and $w^{k} \in T\left(x^{k}\right)$ such that (3.1) and (3.2) hold. This completes the proof.

\section{Preliminary results for iterative sequence}

In what follows, we adopt the following assumptions $\left(A_{1}\right)-\left(A_{4}\right)$ :

$\left(A_{1}\right)$ The solution set $S$ of the problem (1.1) is nonempty (see, for example, [24]). 
$\left(A_{2}\right) f: H \rightarrow(-\infty,+\infty]$ is a proper convex l.s.c function with $X \subset \operatorname{int}(\operatorname{dom} f)$.

$\left(A_{3}\right) T: X \rightarrow 2^{H}$ is a pseudomonotone set-valued mapping with respect to $f$ on $X$ and upper semi-continuous from the weak topology to the weak topology with weakly compact convex values.

$\left(A_{4}\right)$ A fixed relative error tolerance $\sigma \in[0,1)$. Three positive sequences $\left\{\lambda_{k}\right\},\left\{\rho_{k}\right\}$ satisfy (3.5),(3.6) and $\alpha_{k} \in(0,2)$.

Remark 4.1. Since $f$ is a proper convex l.s.c function, $f$ is also weakly l.s.c and continuous over $\operatorname{int}(\operatorname{dom} f)($ see $[26])$.

Remark 4.2. It is obviously that monotone mapping is pseudomonotone with respect to a function $f$, but the converse is not true in general as illustrated by the following set-valued mapping that satisfies $\left(A_{3}\right)$.

EXAMPLE 4.1. Let $H=\mathbb{R}, T: \mathbb{R} \rightarrow 2^{\mathbb{R}}$ be a set-valued mapping defined by:

$$
T(x)=\left\{\begin{array}{cc}
{[x, x+1],} & x \geq 1 \\
1, & x<1 .
\end{array}\right.
$$

Define $f(x)=x, \forall x \in \mathbb{R}$. We have the following conclusions:

(1) $T$ is upper semi-continuous with compact convex values.

(2) $T$ is not a monotone mapping. For example, let $x=2, y=\frac{3}{2}, v=\frac{5}{2} \in T(y)$ and $u=2 \in T(x)$, we have $\langle v-u, y-x\rangle<0$.

(3) $T$ is pseudomonotone mapping with respect to $f$. In fact, $\forall x, y \in \mathbb{R}$ and $\forall u \in T$ $(x)$, if $\langle u, y-x\rangle+f(y)-f(x) \geq 0$, we have $\langle u, y-x\rangle+x-y \geq 0$. So, if $y>x$, we obtain that $\langle v, y-x\rangle \geq y-x>0$ for all $v \geq 1$. By the definition of $T$, we have $\langle v, y-$ $x\rangle+f(y)-f(x) \geq 0$, for all $v \in T(y)$. If $y<x,\langle u, y-x\rangle+x-y \geq 0$ implies that $u \leq$ 1. Since $u \in T(x)$, we have $x \leq 1$ and then $y<1$. By the definition of $T$, we deduce that $v=T(y)=1$ and then $\langle v, y-x\rangle+x-y \geq 0$, for all $v \in T(y)$. That is $\langle v, y-x\rangle+$ $f(y)-f(x) \geq 0, \forall v \in T(y)$. If $y=x$, we always have $\langle v, y-x\rangle+f(y)-f(x) \geq 0$, for all $v$ $\in T(y)$. So, we conclude that $T$ is a pseudomonotone mapping with respect to $f$.

Now, we give some preliminary results for the iterative sequence generated by Algorithm 3.1 in a Hilbert space $H$. First, we state some useful estimates that are direct consequences of the Lemma 2.3.

Theorem 4.1 Under (3.1)-(3.4), if $\mu=\sqrt{1-\left(1-\sigma^{2}\right)^{2}}$, then we have:

(i) $\lambda_{k}(1-\mu)\left\|g^{k}+w^{k}\right\| \leq\left(1-\sigma^{2}\right) \alpha_{k}|| x^{k}-z^{k}\left\|\leq \lambda_{k}(1+\mu)\right\| g^{k}+w^{k} \|$;

(ii) $\left(1-\sigma^{2}\right)\left(\lambda_{k}^{2}\left\|g^{k}+w^{k}\right\|^{2}+\alpha_{k}^{2}\left\|x^{k}-z^{k}\right\|^{2}\right) /\left(2 \alpha_{k} \lambda_{k}\right) \leq\left\langle g^{k}+w^{k}, z^{k}-x^{k}\right\rangle$;

(iii) $\beta_{k} \in\left[\frac{\lambda_{k}\left(1-\sigma^{2}\right)}{2 \alpha_{k}}, \frac{\lambda_{k}(1+\mu)}{\alpha_{k}\left(1-\sigma^{2}\right)}\right]$.

Proof. We apply Lemma 2.3 to $v=g^{k}+w^{k}, u=\alpha_{k}\left(z^{k}-x^{k}\right) / \lambda_{k}$ to get (i) and (ii). For (iii), using first Cauchy-Schwarz inequality and then (i), we get

$$
\beta_{k}=\frac{\left\langle g^{k}+w^{k}, z^{k}-x^{k}\right\rangle}{\left\|g^{k}+w^{k}\right\|^{2}} \leq \frac{\left\|x^{k}-z^{k}\right\|}{\left\|g^{k}+w^{k}\right\|} \leq \frac{\lambda_{k}(1+\mu)}{\alpha_{k}\left(1-\sigma^{2}\right)} .
$$


On the other hand, (ii) implies that

$$
\begin{aligned}
& \beta_{k}=\frac{\left\langle g^{k}+w^{k}, z^{k}-x^{k}\right\rangle}{\left\|g^{k}+w^{k}\right\|^{2}} \\
\geq & \frac{\left(1-\sigma^{2}\right)\left(\lambda_{k}^{2}\left\|g^{k}+w^{k}\right\|^{2}+\alpha_{k}^{2}\left\|x^{k}-z^{k}\right\|^{2}\right)}{2 \alpha_{k} \lambda_{k}\left\|g^{k}+w^{k}\right\|^{2}} \\
= & \frac{\lambda_{k}\left(1-\sigma^{2}\right)}{2 \alpha_{k}}\left[1+\frac{\alpha_{k}^{2}\left\|x^{k}-z^{k}\right\|^{2}}{\lambda_{k}^{2}\left\|g^{k}+w^{k}\right\|^{2}}\right] \\
\geq & \lambda_{k}\left(1-\sigma^{2}\right) /\left(2 \alpha_{k}\right),
\end{aligned}
$$

this leads to (iii).

Remark 4.4. Suppose that $g^{k}+w^{k}=0$ in Step 2. As $-w^{k} \in \partial f\left(x^{k}\right)$, this implies that

$$
\left\langle w^{k}, y-x^{k}\right\rangle+f(y)-f\left(x^{k}\right) \geq 0, \quad \forall y \in X
$$

That is, $x^{k}$ is a solution of problem (1.1). On the other hand, assuming $g^{k}+w^{k} \neq 0$, Theorem 4.1(ii) yields $\left\langle g^{k}+w^{k}, z^{k}-x^{k}\right\rangle>0$. By the pseudomonotonicity of $T$ with respect to $f$, it is easy to see that for all $x^{*} \in S$ ( $S$ denotes the solution set of problem $(1.1))$,

$$
\left\langle w^{k}, x^{*}-x^{k}\right\rangle+f\left(x^{*}\right)-f\left(x^{k}\right) \leq 0, \quad \forall w^{k} \in T\left(x^{k}\right)
$$

Using the fact that $g^{k} \in \partial f\left(x^{k}\right)$, we deduce

$$
0 \geq\left\langle w^{k}, x^{*}-x^{k}\right\rangle+f\left(x^{*}\right)-f\left(x^{k}\right) \geq\left\langle g^{k}+w^{k}, x^{*}-x^{k}\right\rangle .
$$

Thus, the hyperplane $\left\{z \in H:\left\langle g^{k}+w^{k}, z-x^{k}\right\rangle=0\right\}$ strictly separates $z^{k}$ from $S$. The latter is the geometric motivation for the projection step (3.4).

Theorem 4.2. Suppose that $x^{*} \in S$ and the sequence $\left\{\rho_{k}\right\}$ satisfy (3.6), then

$$
\left\|x^{*}-z^{k+1}\right\|^{2} \leq\left\|x^{*}-z^{k}\right\|^{2}-\left(2 / \rho_{k}-1\right)\left\|z^{k+1}-z^{k}\right\|^{2},
$$

and so the sequence $\left\{\left\|x^{*}-z^{k}\right\|^{2}\right\}$ is convergent (not necessarily to 0 ). Moreover,

$$
\sum_{k=0}^{\infty}\left\|z^{k+1}-z^{k}\right\|^{2}<\infty \text { and } \sum_{k=0}^{\infty}\left\|\bar{z}^{k}-z^{k}\right\|^{2}<\infty
$$

Proof. By Step 3, we have

$$
\begin{aligned}
\left\|x^{*}-z^{k+1}\right\|^{2} & =\left\|x^{*}-z^{k}-\rho_{k}\left(\bar{z}^{k}-z^{k}\right)\right\|^{2} \\
& =\left\|x^{*}-z^{k}\right\|^{2}-2 \rho_{k}\left\langle x^{*}-z^{k}, \bar{z}^{k}-z^{k}\right\rangle+\rho_{k}^{2}\left\|\bar{z}^{k}-z^{k}\right\|^{2} \\
& =\left\|x^{*}-z^{k}\right\|^{2}-2 \rho_{k}\left(z^{k}-\bar{z}^{k}, z^{k}-\bar{z}^{k}\right\rangle-2 \rho_{k}\left(\bar{z}^{k}-x^{*}, z^{k}-\bar{z}^{k}\right\rangle+\rho_{k}^{2}\left\|\bar{z}^{k}-z^{k}\right\|^{2} \\
& =\left\|x^{*}-z^{k}\right\|^{2}-2 \rho_{k}\left(\bar{z}^{k}-x^{*}, z^{k}-\bar{z}^{k}\right\rangle+\left(\rho_{k}^{2}-2 \rho_{k}\right)\left\|\bar{z}^{k}-z^{k}\right\|^{2} \\
& =\left\|x^{*}-z^{k}\right\|^{2}-2 \rho_{k}\left(\bar{z}^{k}-x^{*}, z^{k}-\bar{z}^{k}\right\rangle+\left(1-2 / \rho_{k}\right)\left\|z^{k+1}-z^{k}\right\|^{2} .
\end{aligned}
$$

It follows from (4.1) and $x^{*} \in S$ that

$$
x^{*} \in K=\left\{z \in H:\left\langle g^{k}+w^{k}, z-x^{k}\right\rangle \leq 0\right\} .
$$

Since $\bar{z}^{k}=P_{K}\left(z^{k}\right)$, by Proposition 2.1(ii), we deduce that

$$
\left\langle\bar{z}^{k}-x^{*}, z^{k}-\bar{z}^{k}\right\rangle \geq 0 .
$$


So

$$
\left\|x^{*}-z^{k+1}\right\|^{2} \leq\left\|x^{*}-z^{k}\right\|^{2}-\left(2 / \rho_{k}-1\right)\left\|z^{k+1}-z^{k}\right\|^{2} .
$$

By (3.6), we obtain that

$$
0 \leq\left\|x^{*}-z^{k+1}\right\|^{2} \leq\left\|x^{*}-z^{k}\right\|^{2}, \quad \forall k \geq 0 .
$$

Thus, the sequence $\left\{\left\|x^{*}-z^{k}\right\|^{2}\right\}$ is convergent. Let $L_{\infty}$ be the limit of $\left\{\left\|x^{*}-z^{k}\right\|^{2}\right\}$.

Now, we prove that (4.3) holds. It follows from (3.6) and (4.2) that

$$
0 \leq\left(2 / R_{2}-1\right)\left\|z^{k+1}-z^{k}\right\|^{2} \leq\left(2 / \rho_{k}-1\right)\left\|z^{k+1}-z^{k}\right\|^{2} \leq\left\|x^{*}-z^{k}\right\|^{2}-\left\|x^{*}-z^{k+1}\right\|^{2}(4.21)
$$

(4.4) implies that

$$
0 \leq\left(2 / R_{2}-1\right) \sum_{k=0}^{\infty}\left\|z^{k+1}-z^{k}\right\|^{2} \leq \sum_{k=0}^{\infty}\left[\left\|x^{*}-z^{k}\right\|^{2}-\| x^{*}-z^{k+1}||^{2}\right]=\left\|x^{*}-z_{0}\right\|^{2}-L_{\infty},
$$

and then $\sum_{k=0}^{\infty}\left\|z^{k+1}-z^{k}\right\|^{2}<\infty$ holds. On the other hand, $0 \leq R_{1}\left\|\bar{z}^{k}-z^{k}\right\| \leq \rho_{k}\left\|\bar{z}^{k}-z^{k}\right\|=\left\|z^{k+1}-z^{k}\right\|$, so that we obtain $\sum_{k=0}^{\infty}\left\|\bar{z}^{k}-z^{k}\right\|^{2}<\infty$. This completes the proof.

Theorem 4.3. Suppose that assumption $\left(A_{4}\right)$ holds, then there exists some constant $\zeta$ $>0$ such that

$$
\left\langle z^{k}-x^{k}, g^{k}+w^{k}\right\rangle \geq \zeta\left\|g^{k}+w^{k}\right\|^{2} .
$$

Proof. By Theorem 4.1(ii), we have

$$
\begin{aligned}
\left\langle g^{k}+w^{k}, z^{k}-x^{k}\right\rangle & \geq\left(1-\sigma^{2}\right)\left(\lambda_{k}^{2}\left\|g^{k}+w^{k}\right\|^{2}+\alpha_{k}^{2}\left\|x^{k}-z^{k}\right\|^{2}\right) /\left(2 \alpha_{k} \lambda_{k}\right) \\
& \geq\left(1-\sigma^{2}\right) \lambda_{k}\left\|g^{k}+w^{k}\right\|^{2} /\left(2 \alpha_{k}\right) .
\end{aligned}
$$

Since $\lambda_{k} \in\left[\lambda_{1}, \lambda_{2}\right]$ and $\alpha_{k} \in(0,2)$,

$$
\left\langle g^{k}+w^{k}, z^{k}-x^{k}\right\rangle \geq \frac{\lambda_{1}\left(1-\sigma^{2}\right)}{4}\left\|g^{k}+w^{k}\right\|^{2} .
$$

This completes the proof.

Theorem 4.4. Suppose that assumption $\left(A_{4}\right)$ holds, then

$$
\lim _{k \rightarrow \infty}\left\|g^{k}+w^{k}\right\|=0
$$

Proof. It follows from (3.4) and (4.5) that, for all $k$ for which $g^{k}+w^{k} \neq 0$,

$$
\begin{aligned}
\left\|z^{k}-z^{k}\right\| & =\left\|\beta_{k}\left(g^{k}+w^{k}\right)\right\| \\
& =\left\langle g^{k}+w^{k}, z^{k}-x^{k}\right\rangle /\left\|g^{k}+w^{k}\right\| \\
& \geq \zeta\left\|g^{k}+w^{k}\right\|,
\end{aligned}
$$

which clearly also holds for $k$ satisfying $g^{k}+w^{k}=0$. By (4.3) and (4.7), we have

$$
\lim _{k \rightarrow \infty}\left\|g^{k}+w^{k}\right\|=0 .
$$

This completes the proof.

\section{Convergence analysis}

We now study the convergence of Algorithm 3.1. 
Theorem 5.1. Suppose that the sequence $\left\{x^{k}\right\}$ generated by Algorithm 3.1 is finite. Then, the last term is a solution of the problem (1.1).

Proof. If the sequence is finite, then it must stop at Step 2 for some $x^{k}$. In this case, we have $g^{k}+w^{k}=0$. By Remark 4.4, we know that $x^{k} \in X$ is a solution of problem (1.1). This completes the proof.

From now on, we assume that the sequence $\left\{x^{k}\right\}$ generated by Algorithm 3.1 is infinite and so is the sequence $\left\{z^{k}\right\}$.

Theorem 5.2. Let $\left\{x^{k}\right\}$ and $\left\{z^{k}\right\}$ be sequences generated by Algorithm 3.1 under assumptions $\left(A_{1}\right)-\left(A_{4}\right)$. Then, $\left\{x^{k}\right\}$ and $\left\{z^{k}\right\}$ are bounded. Moreover, $\left\{x^{k}\right\}$ and $\left\{z^{k}\right\}$ have the same weak accumulation points.

Proof. It follows from Theorem 4.2 that the sequence $\left\{z^{k}\right\}$ is bounded. Using Theorem 4.4 and Theorem 4.1(i), we know that

$$
\lim _{k \rightarrow \infty}\left\|z^{k}-x^{k}\right\|=0,
$$

and so

$$
\lim _{k \rightarrow \infty}\left(z^{k}-x^{k}\right)=0 .
$$

By the boundedness of the sequence $\left\{z^{k}\right\}$, we obtain that the sequence $\left\{x^{k}\right\}$ is bounded. Moreover, (5.1) implies that the two sequences $\left\{x^{k}\right\}$ and $\left\{z^{k}\right\}$ have the same weak accumulation points. This completes the proof.

Theorem 5.3. Suppose that assumptions $\left(A_{1}\right)-\left(A_{4}\right)$ hold. Then, every weak accumulation point of the sequence $\left\{x^{k}\right\}$ generated by Algorithm 3.1 is a solution of problem (1.1). Moreover, every weak accumulation point of the sequence $\left\{z^{k}\right\}$ generated by Algorithm 3.1 is also a solution of problem (1.1)

Proof. Let $\hat{x}$ be a weak accumulation point of $\left\{x^{k}\right\}$, we can extract a subsequence that weakly converges to $\hat{x}$. Without loss of generality, let us suppose that $\lim _{k \rightarrow \infty} x^{k}=\hat{x}$. It is obvious that $\hat{x} \in X$. By (5.1), we have $\lim _{k \rightarrow \infty} z^{k}=\hat{x}$.

Now, we prove each weak accumulation point of $\left\{x^{k}\right\}$ is a solution of the problem (1.1). By $\bar{z}^{k}=z^{k}-\beta_{k}\left(g^{k}+w^{k}\right)$ and $g^{k} \in \partial f\left(x^{k}\right)$, we deduce that for each $y \in X$,

$$
\left\langle w^{k}, y-x^{k}\right\rangle+f(y)-f\left(x^{k}\right) \geq \frac{1}{\beta_{k}}\left\langle z^{k}-\bar{z}^{k}, y-x^{k}\right\rangle,
$$

where $w^{k} \in T\left(x^{k}\right)$. It follows that

$$
\sup _{w^{k} \in T\left(x^{k}\right)}\left[\left\langle w^{k}, y-x^{k}\right\rangle+f(y)-f\left(x^{k}\right)\right] \geq \frac{1}{\beta_{k}}\left\langle z^{k}-\bar{z}^{k}, y-x^{k}\right\rangle, \quad \forall y \in X .
$$

By Theorem 4.1(iii) and $\left(A_{4}\right)$, we have

$$
0<\frac{\alpha_{k}\left(1-\sigma^{2}\right)}{\lambda_{k}(1+\mu)} \leq \frac{1}{\beta_{k}} \leq \frac{2 \alpha_{k}}{\lambda_{k}\left(1-\sigma^{2}\right)} \leq \frac{4}{\lambda_{1}\left(1-\sigma^{2}\right)} .
$$

For each fixed $y \in X$, (5.3) implies that

$$
\left|\frac{1}{\beta_{k}}\left\langle z^{k}-\bar{z}^{k}, y-x^{k}\right\rangle\right| \leq \frac{4}{\lambda_{1}\left(1-\sigma^{2}\right)}\left\|z^{k}-\bar{z}^{k}\right\||| y-x^{k}|| .
$$


It follows from (5.4), (4.3) and the boundedness of $\left\{x^{k}\right\}$ that

$$
\lim _{k \rightarrow \infty}\left[\frac{1}{\beta_{k}}\left\langle z^{k}-\bar{z}^{k}, y-x^{k}\right\rangle\right]=0 .
$$

On the other hand, by assumptions $\left(A_{2}\right)$ and $\left(A_{3}\right)$, Lemma 2.2 implies that $V(x)$ : = $\sup _{w \in T(x)}[\langle w, y-x\rangle+f(y)-f(x)]$ is a weak upper semi-continuous function. Using the fact $\lim _{k \rightarrow \infty} x^{k}=\hat{x}$ (weakly), we have

$$
V(\hat{x}) \geq \lim _{k \rightarrow \infty} V\left(x^{k}\right)
$$

and so

$$
\sup _{w \in T(\hat{x})}[\langle w, y-\hat{x}\rangle+f(\gamma)-f(\hat{x})] \geq \lim _{k \rightarrow \infty} \sup _{w^{k} \in T\left(x^{k}\right)}\left[\left\langle w^{k}, y-x^{k}\right\rangle+f(\gamma)-f\left(x^{k}\right)\right] .
$$

By (5.2), (5.5) and (5.6),

$$
\sup _{w \in T(\hat{x})}[\langle w, y-\hat{x}\rangle+f(y)-f(\hat{x})] \geq 0, \quad \forall y \in X .
$$

Using assumption $\left(A_{3}\right)$, we know that there exists $\hat{w} \in T(\hat{x})$ such that

$$
\langle\hat{w}, y-\hat{x}\rangle+f(y)-f(\hat{x}) \geq 0, \quad \forall y \in X .
$$

That is, $\hat{x} \in X$ is a solution of problem (1.1). This completes the proof.

The following uniqueness argument just given closely follows the one of Martinet [27] (also see Rockafellar [4]), but we give the proof for the convenience of the reader.

Theorem 5.4. Suppose that assumptions $\left(A_{1}\right)-\left(A_{4}\right)$ hold. Then, the sequence $\left\{z^{h}\right\}$ generated by Algorithm 3.1 has a unique weak accumulation point, thus, $\left\{z^{k}\right\}$ is weakly convergent and so does the sequence $\left\{x^{k}\right\}$.

Proof. For each $x^{*} \in S$, it follows from Theorem 4.2 that the sequence $\left\{\left\|z^{k}-x^{*}\right\|^{2}\right\}$ converges (not necessarily to 0 ). Now, we prove that the sequence $\left\{z^{k}\right\}$ has a unique weak accumulation point and so does the sequence $\left\{x^{k}\right\}$. Existence of weak accumulation points of $\left\{z^{k}\right\}$ follows from Theorem 5.2. Let $\hat{z}$ and $\bar{z}$ be two weak accumulation points of $\left\{z^{k}\right\}$ and $\left\{z^{k_{j}}\right\},\left\{z^{k_{i}}\right\}$ be two subsequences of $\left\{z^{k}\right\}$ that weakly converge to $\hat{z}, \bar{z}$ respectively. By Theorem 5.3, we know that $\hat{z}, \bar{z} \in S$. Then, the sequences $\left\{\left\|z^{k}-\hat{z}\right\|^{2}\right\}$ and $\left\{\left\|z^{k}-\bar{z}\right\|^{2}\right\}$ are convergent. Let $\xi=\lim _{k \rightarrow \infty}\left\|z^{k}-\hat{z}\right\|^{2}, \eta=\lim _{k \rightarrow \infty}\left\|z^{k}-\bar{z}\right\|^{2}$ and $\gamma=\|\hat{z}-\bar{z}\|^{2}$. Then,

$$
\left\|z^{k_{j}}-\bar{z}\right\|^{2}=\left\|z^{k_{j}}-\hat{z}\right\|^{2}+\|\hat{z}-\bar{z}\|^{2}+2\left\langle z^{k_{j}}-\hat{z}, \hat{z}-\bar{z}\right\rangle
$$

and

$$
\left\|z^{k_{i}}-\hat{z}\right\|^{2}=\left\|z^{k_{i}}-\bar{z}\right\|^{2}+\|\hat{z}-\bar{z}\|^{2}+2\left\langle z^{k_{i}}-\bar{z}, \bar{z}-\hat{z}\right\rangle .
$$

Take limit in (5.7) as $j \rightarrow \infty$ and (5.8) as $i \rightarrow \infty$, observing that the inner products in the right hand sides of (5.7) and (5.8) converge to 0 because $\hat{z}, \bar{z}$ are weak limits of $\left\{z^{k_{i}}\right\},\left\{z^{k_{i}}\right\}$ respectively, and get, using the definitions of $\xi, \eta, \gamma$,

$$
\begin{gathered}
\xi=\eta+\gamma, \\
\eta=\xi+\gamma .
\end{gathered}
$$


From (5.9) and (5.10), we get $\xi-\eta=\gamma=\eta-\xi$, which implies $\gamma=0$, i.e., $\hat{z}=\bar{z}$. It follows that all weak accumulation points of $\left\{z^{k}\right\}$ coincide, i.e., $\left\{z^{k}\right\}$ is weakly convergent. This completes the proof.

\section{Acknowledgements}

The authors are grateful to Professor Kun-Quan Lan and the referees for their valuable comments and suggestions leading to the improvement of this paper. This work was supported by the National Natural Science Foundation of China (10671135), the Specialized Research Fund for the Doctoral Program of Higher Education (20105134120002), the Application Foundation Fund of Sichuan Technology Department of China (2010JY0121), the NSF of Sichuan Education Department of China (09ZA091).

\section{Author details}

${ }^{1}$ Department of Mathematics, Sichuan Normal University, Chengdu, Sichuan 610066, P. R. China ${ }^{2}$ Department of Mathematics, Sichuan University, Chengdu, Sichuan 610064, P. R. China

\section{Authors' contributions}

Both authors contributed equally to this work. All authors read and approved the final manuscript.

\section{Competing interests}

The authors declare that they have no competing interests.

Received: 8 November 2010 Accepted: 25 July 2011 Published: 25 July 2011

\section{References}

1. Cohen G: Nash equilibria: gradient and decomposition algorithms. Large Scale Syst 1987, 12:173-184.

2. Konnov I: A combined relaxation method for a class of nonlinear variational inequalities. Optimization 2002, 51:127-143.

3. Panagiotopoulos $P$, Stavroulakis G: New types of variational principles based on the notion of quasidifferentiablity. Acta Mech 1994, 94:171-194.

4. Rockafellar RT: Monotone operators and the proximal point algorithm. SIAM J Control Optim 1976, 14:877-898.

5. Tseng P: Applications of a splitting algorithm to decomposition in convex programming and variational inequalities. SIAM J Control Optim 1991, 29:119-138.

6. Xia FQ, Huang NJ: A projected subgradient method for solving generalized mixed variational inequalities. Oper Res Lett 2008, 36:637-642.

7. Crouzeix JP: Pseudomonotone variational inequality problems: existence of solutions. Math Program 1997, 78:305-314.

8. Danniilidis A, Hadjisavvas N: Coercivity conditions and variational inequalities. Math Program 1999, 86:433-438.

9. Yao JC: Multivalued variational inequalities with K-pseudomonotone operators. J Optim Theory Appl 1994 , 83:391-403.

10. Facchinei F, Pang JS: Finite Dimensional Variational Inequalities and Complementarity Problems. Springer-Verlag, New York; 2003.

11. He YR: A new double projection algorithm for variational inequalities. J Comput App/ Math 2006, 185:166-173.

12. Harker PT, Pang JS: Finite dimensional variational inequality and nonlinear complementarity problems: a survey of theory, algorithms and applications. Math Programm 1990, 48:161-220.

13. Marcotte P: Application of Khobotov's algorithm to variational inequalities and network equilibrium. Inf Syst Oper Res 1991, 29:258-270.

14. Solodov MV, Svaiter BF: A new projection method for variational inequality problems. SIAM J Control Optim 1999, 37:765-776.

15. Cohen G: Auxiliary problem principle extended to variational inequalities. J Optim Theory Appl 1988, 49:325-333.

16. Salmon G, Strodiot JJ, Nguyen VH: A bundle method for solving variational inequalities. SIAM J Optim 2004, 14:869-893.

17. Eckstein J, Bertsekas DP: On the Douglas-Rachford splitting method and the proximal point algorithm for maximal monotone operators. Math Program 1992, 55:293-318.

18. Lions PL, Mercier B: Splitting algorithms for the sum of two nonlinear operators. SIAM J Numer Anal 1979, 16:964-979.

19. Bauschke HH, Combettes PL, Reich S: The asymptotic behavior of the composition of two resolvents. Nonlinear Anal 2005, 60:283-301.

20. Gabay D: Applications of the method of multipliers to variational inequalities. In Augmented Lagrangian Methods: Applications to the Solution of Boundary Value Problems. Volume Chap IX. Edited by: Fortin M, Glowinski R. North-Holland, Amsterdam; 1983:299-340.

21. Tseng P: A modified forward-backward splitting method for maximal monotone mapping. SIAM I Control Optim 2000, 38:431-446.

22. Polyak BT: Introduction to Optimization. Optimization Software, New York; 1987.

23. Ding XP, Tan KK: A minimax inequality with application to the existence of equilibrium point and fixed point theorems. Colloquium Math 1992, 63:233-247.

24. Aubin JP, Ekeland I: Applied Nonlinear Analysis. Wiley, New York; 1984.

25. Solodov MV, Svaiter BF: A unified framework for some inexact proximal point algorithms. Numer Funct Anal Optim 2001, 22:1013-1035.

26. Ekeland I, Temam R: Convex Analysis and Variational Inequalities. North-Holland, Amsterdam; 1976. 
27. Martinet B: Régularisation d'inéquations variationelles par approximations successives. Rev Francaise Informat Recherche Opérationnelle 1970, 4:154-158.

doi:10.1186/1029-242X-2011-27

Cite this article as: Xia and Zou: A projective splitting algorithm for solving generalized mixed variational inequalities. Journal of Inequalities and Applications 2011 2011:27.

Submit your manuscript to a SpringerOpen ${ }^{\odot}$ journal and benefit from:

- Convenient online submission

- Rigorous peer review

- Immediate publication on acceptance

- Open access: articles freely available online

- High visibility within the field

- Retaining the copyright to your article

Submit your next manuscript at $\boldsymbol{\nabla}$ springeropen.com 